\title{
Apparent Superluminal Speeds in Evanescent Fields, Quantum Tunnelling and Quantum Entanglement
}

\author{
Arne Bergstrom* \\ B\&E Scientific Ltd, Seaford BN25 4PA, United Kingdom \\ *Corresponding author: arne.bergstrom@physics.org
}

Received January 22, 2015; Revised January 25, 2015; Accepted January 27, 2015

\begin{abstract}
Three cases of wave propagation involving possible superluminal speeds are discussed. The picture that emerges is that the actual propagation velocity of the wave front never exceeds the speed of light in vacuum. However, once the wave front has reached some distant point in space, then propagation may actually seem to take place along this wave with superluminal speed, yet involving no conflict with special relativity. Quantum entanglement - Einstein's "spooky action at a distance" - is one famous, and now experimentally verified example of propagation at such apparent superluminal speed, but which is here explained within the framework of special relativity. This then at the same time also leads to a deeper understanding of the limitation of the recently proposed clock-hypothesis in special relativity, and also provides an illustration of the mechanism involved in wave-particle duality.
\end{abstract}

Keywords: superluminal group velocities, special relativity valid, limited validity of clock hypothesis

Cite This Article: Arne Bergstrom, "Apparent Superluminal Speeds in Evanescent Fields, Quantum Tunnelling and Quantum Entanglement.” International Journal of Physics, vol. 3, no. 1 (2015): 40-44. doi: 10.12691/ijp-3-1-7.

\section{Introduction}

From Maxwell's equations follow that electromagnetic waves in vacuum propagate with the speed of light. According to Einstein's theory of special relativity, the speed of light would also seem to be the maximum speed with which information can travel, otherwise we would seem to be at risk to get involved into problems about cause and effect with regard to reference systems with different relativistic velocities.

However, it now also seems clear - even if maybe not fully understood - that some physical phenomena would seem to transcend the limitation thus imposed by the speed of light. One such example is quantum entanglement [1,2] - what Einstein called 'spukhafte Fernwirkung' ('spooky action at a distance'), and which will here be discussed at some length in Sec. 4 below. Before that, tunnelling in quantum mechanics, which is another possible example involving such superluminal velocities, will be discussed in Sec. 3. A macroscopic equivalent to quantum tunnelling is the evanescent field at total internal reflection, which will here now first be discussed.

Wikipedia has extensive articles on "Faster-than-light" and "Quantum_entanglement", which are recommended as sources for further references in this vibrant field.

\section{Superluminal Speed of the Evanescent Field?}

One practical application of the evanescent field was the optical touch key - a forerunner to the touch keys in present-day iPads and the like - which was patented [3] in 1978 and marketed [4] by OptiSensor AB. In this type of non-mechanical key, the finger interfered with the evanescent field at total internal reflection in a rectangular prism as illustrated in Figure 1, Figure 2a and Figure 2b.

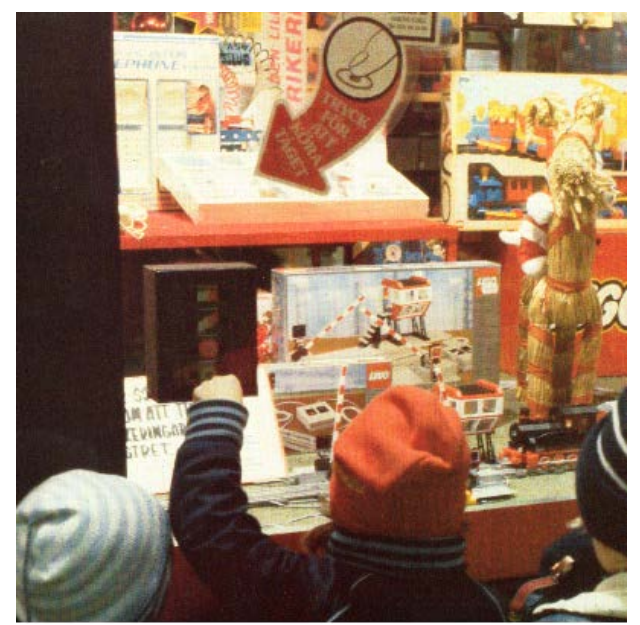

Figure 1. OptiSensor's optical touch key operating a toy train at Stor och Liten toyshop in Stockholm at Christmas time in the early 1980s. The black box is temporarily glued in optical contact to the inside of the window and sends infrared light beams that experience total internal reflection in the outer window surface, but where the evanescent field immediately outside the window surface can be dampened by touching the window with a finger and thus control the train. The finger tip of the child interacts with the evanescent field outside the glass with superluminal group velocity as explained in Sec. 3 


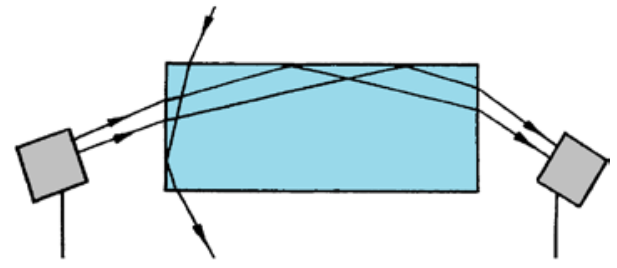

Figure 2a. A rectangular glass prism has the property that a light ray from, $e g$, a LED to the left entering a side face of the prism can only exit through the opposite side face, where it is being detected (or through the same side face after reflections). No matter how many successive reflections may take place in a rectangular prism, the light ray will always experience total internal reflection in the other side faces. Similarly, external light will always be totally reflected in the side faces and cannot reach the detector. Using optical glass with a slightly higher refractive index, this separation between the ray systems can be maintained even in water (rain will not disturb the operation)

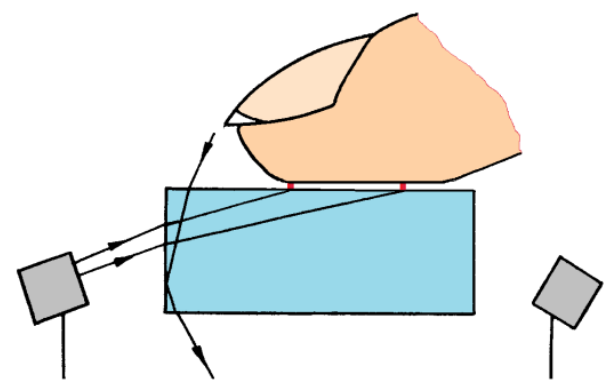

Figure 2b. However, at total internal reflection an evanescent field (red, range exaggerated in the figure) extends into the outer medium and can be absorbed there by a finger. This thus causes part of the totally reflected light to vanish and not reach the detector. In Figure 1 the prism was attached in optical contact with the window, but since all surfaces are rectangular, the same separation between the orthogonal ray systems was maintained, i e external light will not interfere with the operation

In one application, the optical touch key was used on shop windows, on the inside of which the keyboard was semi-permanently glued in optical contact, as shown in Figure 1. Displays in the shop window could in this way be activated by touching the outside of the window (Figures $2 \mathrm{a}$ and $2 \mathrm{~b}$ explain the role of the evanescent field in this case in somewhat more detail).

Total internal reflection of this kind has also been demonstrated with microwaves in a block of paraffin, and where the range of the evanescent field outside the prism then can be of the order of centimetres.

When studying total internal reflection of the type occurring in the optical touch key described above, it seemed difficult at the time to understand the behaviour of a photon that had just experienced total internal reflection at the surface inside the glass (or paraffin), and had thus now moved some distance inside the glass back away from the surface. How could - from a pedestrian point of view - such a photon retrace its path after a while and instead decide to disappear just because someone had in the meantime put a finger within reach of the evanescent field on the outside of the surface and at some distance away from it? It seemed difficult to understand this in any other way than that the evanescent field outside the glass or paraffin must have propagated with infinite speed. Only in that way would it seem to have got the information of a possible outside breach some distance away of the total internal reflection and been able to convey this information to the photon already when it was at the surface, and thus allow the reflection not to occur.
It is interesting to see how diffuse, unspecified clutter of thoughts like this can precede new insights or discoveries, just like, $e g$, a similar case of the clutter of thoughts relating to dark energy/matter before it was known to exist at all $[5,6,7]$. Or, in the words of Jorge Luis Borges [8]: "the word 'precursor' ... should be cleansed of all connotations of polemic or rivalry. The fact is that every writer creates his own precursors. His work modifies our conception of the past, as it will modify the future."

The main factor in the progress in the case of superluminal velocities has been the advent of methods to measure extremely short time intervals in the laboratory. This has now thus finally made it possible, notably in the experiments by Steinberg, Kwiat, and Chiao [9], to study such possible superluminal velocities experimentally. In these experiments a tunnelled photon was measured to have propagated with a velocity 1.7 times the speed of light. This will be further discussed in Sec. 3 below.

The existence of a possible superluminal component at total internal reflection has been described in earlier texts, but then historically considered not to be very relevant but mainly to be a relic of a non-quantum description. However, since the effect occurs also in large systems such as in microwave cavities [10] or a large block of paraffin, it needs to be taken seriously. Whatever effects Maxwell's equations predict for this kind of behaviour must thus be understood to be real and not to be artefacts just due to the use of Maxwell's equations on microscopic systems outside their range of validity.

Detailed theoretical calculations by Barbero, Hernández-Figueroa, and Recami [10], of evanescent fields in undersized waveguides show ( $c f$ Figure 3a and Figure $3 b$ ) the onset of the electromagnetic field to propagate with the speed of light, and then first to give a tiny Sommerfeld precursor followed by a larger Brillouin precursor before the main wave arrives. In this main wave, group velocities may very well exceed the speed of light, but will never be able to overtake the start of the wave. However, superluminal group velocities of this kind are not considered to be able to propagate information, which would thus exclude, e g, superluminal communication.

\section{Superluminal Speed at Quantum Tunnelling?}

Chiao, Kwiat, and Steinberg have further discussed their results mentioned above in an article [11] in Scientific American. In this article, the authors explain their results by picturing two tortoises competing to reach the other end of a field, each tortoise representing the wave packet of a particle in their experiment as illustrated in Figure 4.

One of the tortoises has to pass through a barrier, where it splits into two: one small tortoise that passes through the barrier whereas the rest of the tortoise gets reflected. If the tortoises crawl with the same speed, the nose of the tortoise that had no obstacle and the nose of the small tortoise will both pass the finishing line at the same time.

But the point is that if we detect the arrival of the tortoises at the finishing line not by their noses ( $i e$ the somewhat hard-to-measure front of the minute Sommerfeld precursor at $t_{0}$ in Figure $3 \mathrm{~b}$ ) but instead by 
the top of their backs ( $i e$ the maximum of the wave packets as in Figure 3c), then the smaller tortoise will seem to be ahead as illustrated in Figure 4.

Interpreted in this way, there would thus no longer be any real superluminal speed involved in this experiment nor in the related optical touch key discussed in Sec. 2 only a superluminal group velocity. Since the wave packet corresponding to the smaller tortoise has shrunk in length due to its superluminal group velocity, then as a consequence its maximum has thus been shifted forwards.

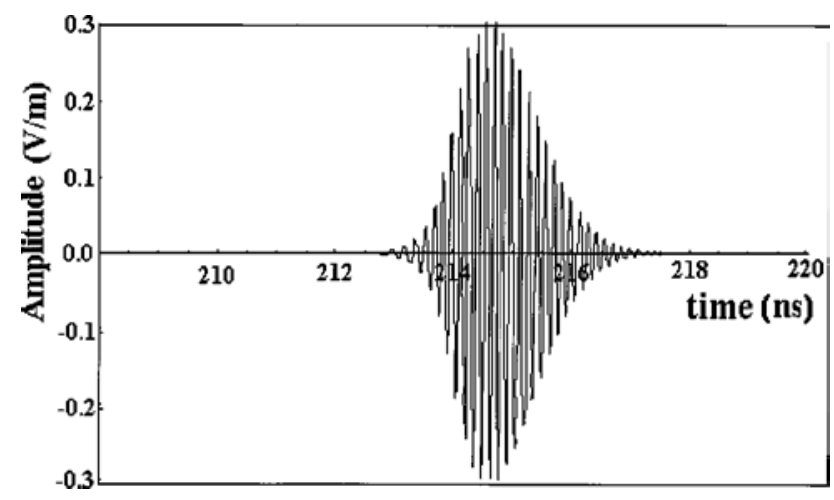

Figure 3a. A propagating wave packet in an undersized waveguide (from Barbero, Hernández-Figueroa and Recami [10] (reprinted with permission from assocpub@aps.org)

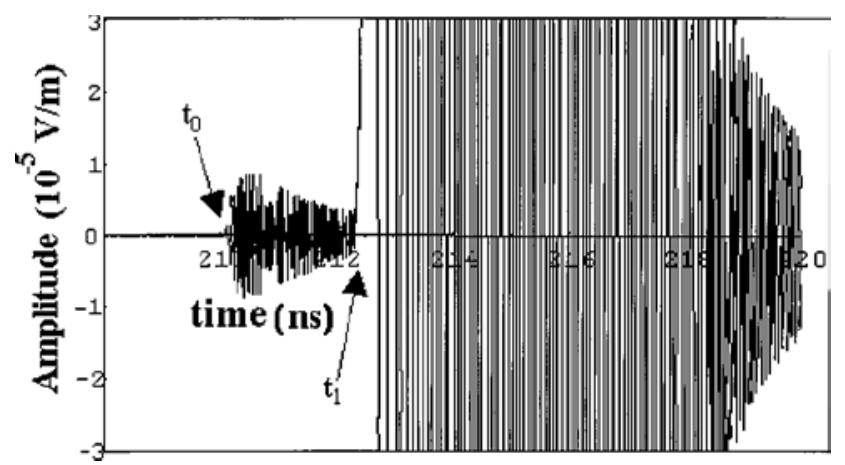

Figure 3b. The same wave packet as in Figure 3a with the same time scale but with the amplitude scale enlarged by a factor of $10^{4}$, showing the tiny Sommerfeld precursor at $t_{0}$ preceding the somewhat larger Brillouin precursor at $t_{1}$ before the bulk of the wave packet arrives [10] (reprinted with permission from assocpub@aps.org)

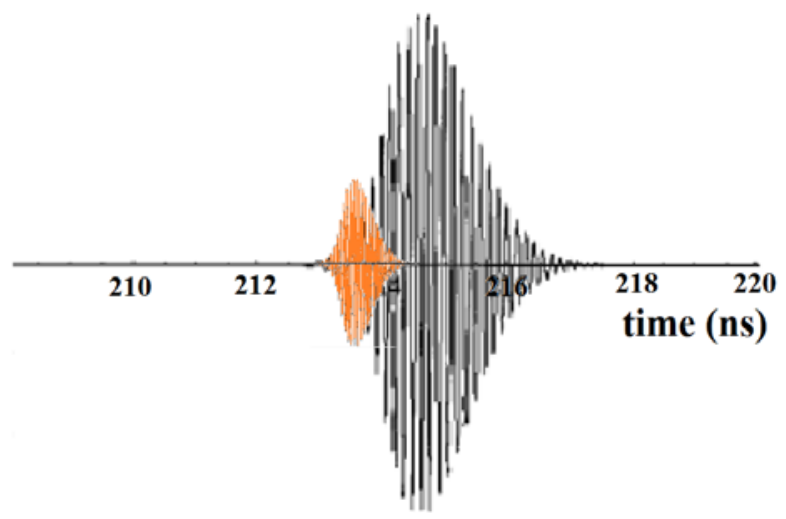

Figure 3c. Comparison between wave packets with different group velocities. The group velocity in the smaller, red wave packet is higher than in the main wave packet. Despite the precursors for both wave packets in this case arriving at the same time, the maximum of the red wave packet arrives before the maximum of the main wave packet due to the compression caused by its higher group velocity. This thus appears as a superluminal velocity as is also illustrated in Figure 4

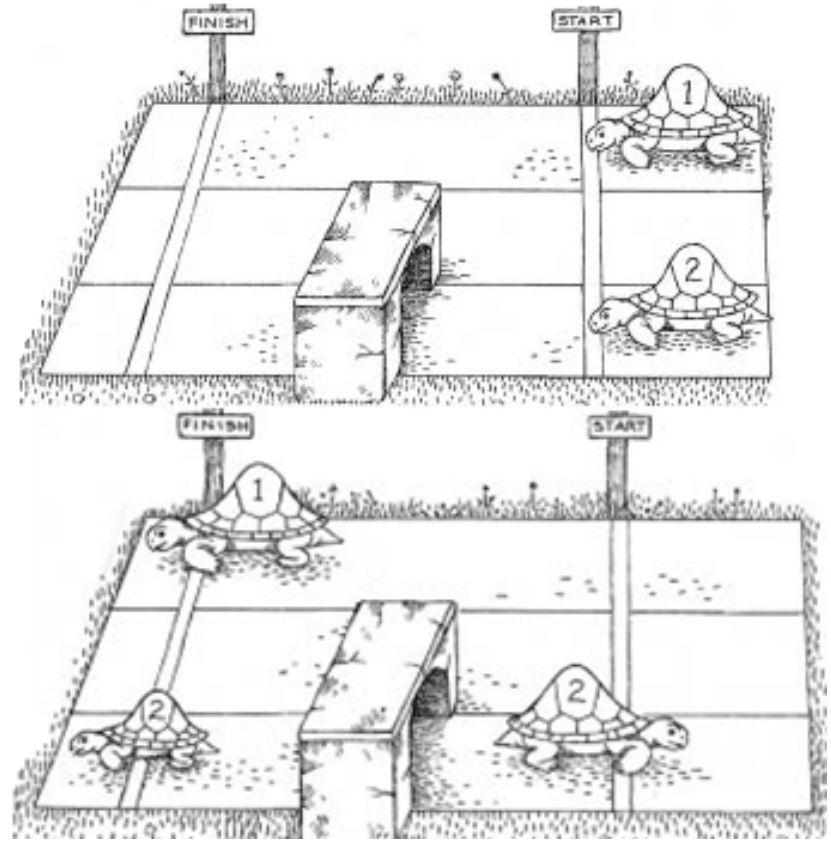

Figure 4. Superluminal group velocity illustrated [11] by two tortoises competing to reach the other end of a field. A barrier splits one of the tortoises into two: one part being reflected by the barrier and the other part being transmitted through it. Even though the tortoises both crawl with the same speed $c$, and their noses thus reach the finishing line at the same time, the top of the back of the smaller tortoise is ahead compared to the top of the back of the bigger tortoise, illustrating how group velocities in a barrier experiment thus can exceed the velocity $c$ (reprinted with permission from Scientific American and Patricia J. Wynne, Wynneart@aol.com)

\section{Superluminal Speed at Quantum Entanglement?}

As apparent from Einstein's comment cited above of 'spukhafte Fernwirkung', quantum entanglement has been a controversial subject for a long time.

Experiments showing quantum entanglement have recently been performed with photons, electrons, molecules including large molecules like buckyballs [12] $\left(\mathrm{C}_{60}\right)$, and even small diamonds [13]. Of particular interest in connection to the question of superluminal speeds are experiments showing entanglement between quanta separated by large distances [14], and where thus some effects must be interpreted to have propagated with superluminal speed. This would thus seem to be in conflict with basic principles of special relativity.

However, concerning such seemingly superluminal velocities in connection with entangled photons, or other entangled particles moving essentially at light speed, these superluminal velocities can be understood as a relativistic effect. This will be discussed in the following, and it seems especially enlightening to discuss this question in relation to the assumed clock-hypothesis in special relativity.

The clock-hypothesis has comparatively recently [15] been introduced into special relativity as a new postulate, and states that accelerated motion of a system does not affect the passage of time in that system compared to a corresponding non-accelerating system. The clockhypothesis now seems to be essentially generally accepted [16] as a universal principle in special relativity, and 
claiming to prohibit time to be dependent on acceleration in all situations.

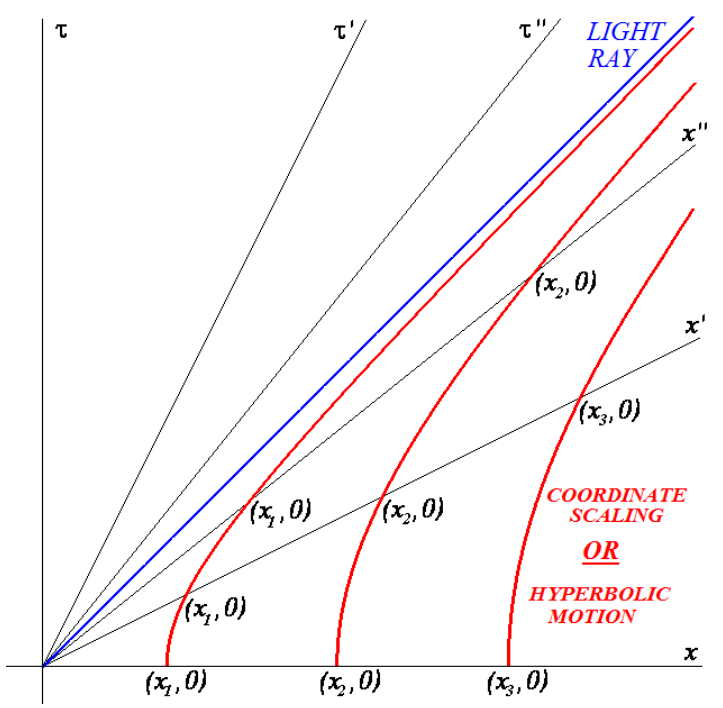

Figure 5. Minkowski diagram showing how the space and time axes in moving systems (primed) relate to the corresponding axes in a stationary system $x \tau$. Spacetime points $\left(x_{\mathrm{i}}, 0\right)$ transform as hyperbolas (red) for increasing relativistic velocities, and which coincide with the hyperbolic spacetime trajectories for particles in constant proper acceleration. Time shown by a clock moving along such a hyperbolic spacetime trajectory will thus appear slowed-down or even frozen to a stationary observer. Light is the special limiting case when $x_{\mathrm{i}}=0$ (blue). Then all points $(x, x)$ of the light ray in the stationary system coalesce into one point $(0,0)$ in the co-moving system - thus making quantum entanglement understandable even between entangled photons separated by large distances in the stationary system as illustrated in Figure 6

However, a problem is then that the clock hypothesis would seem to be in conflict with acceleration-gravitation equivalence, since gravitation is well documented to affect the passage of time, most spectacularly in black holes and accelerations should thus be expected to do so too in corresponding cases. Furthermore, such a principle requiring time always to be independent of accelerations would obviously also require time in a system in hyperbolic spacetime motion to be unaffected by this particular type of acceleration and thus just tick on as it would if there were no acceleration.

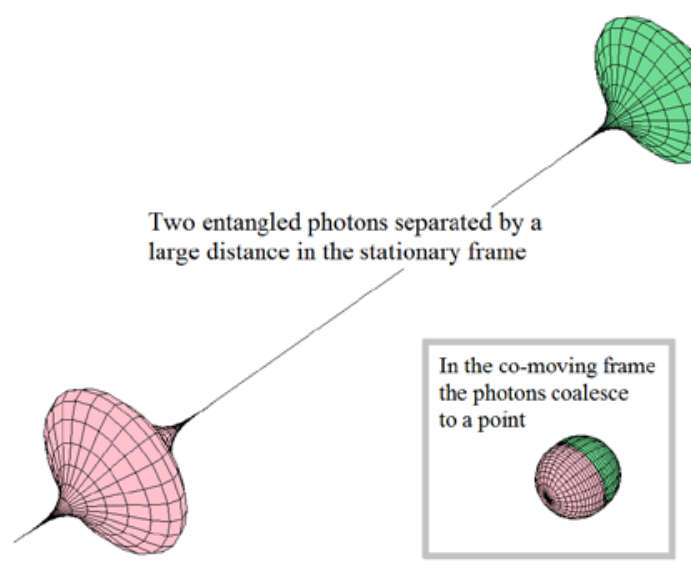

Figure 6. Schematic illustration of how two entangled photons in the stationary system are observed in their own system to be simultaneously at the same point as discussed in Figure 5. Their entire wave functions in the stationary system transform into a point in their own system, which explains how measurement of the state of one of them is instantaneously transferred to the other one
If so, a crucial problem [17] is then that the scaling of the axes at different relativistic velocities in the Minkowski spacetime diagram is actually defined by just such hyperbolas. A spacetime point $(x, 0)$ specifically describes such a hyperbola in the Minkowski diagram for coordinate systems with different relative velocities as illustrated in Figure 5. For a specific choice of space coordinate, say $x_{\mathrm{i}}$, such a hyperbola thus represents a constant, unchanging proper time $\tau^{\prime}=\tau^{\prime}{ }^{\prime}=\tau^{\prime}{ }^{\prime}{ }^{\prime} \ldots=0$ along such a hyperbola at different relative velocities, $i e$ at the specific acceleration described by such hyperbolic motion in the stationary system. This would thus be in apparent conflict with time just ticking on independently of the acceleration involved, as stipulated by the clockhypothesis.

Hence, even though [17] time in special relativity can be derived to be independent of accelerations in agreement with the clock-hypothesis, the properties of hyperbolic motion imply that, in the special case of such motion, time will nevertheless be slowed-down or maybe even frozen. Such motion is also the type of motion occurring in the acceleration-gravitation equivalence, and should thus also for that reason be expected to involve slowed-down or even frozen time.

The resolution of this seeming paradox lies in the fact that hyperbolas in the Minkowski diagram appear in two different, but still identical forms [17] as illustrated in Figure 5:

1. describing the spacetime trajectories for motion at constant proper acceleration (hyperbolic motion), and

2. describing constant proper time at different relativistic velocities (the hyperbolic coordinate scales in the Minkowski diagram).

Time may thus in general be unaffected by accelerations as specified by the clock-hypothesis. However, there is then an exception in the form of a special case: A system in hyperbolic motion with respect to a stationary reference system will still, due to the special scaling properties of this particular type of motion [17], nevertheless be affected by time dilation in this special type of accelerations, and time may even appear frozen in such a system when observed from the stationary system.

These properties of hyperbolic motion are important since they provide a framework to determine the properties of light in the limit when the hyperbolic trajectories approach light rays as depicted in Figure 5. This has immediate relevance to the problem of the seemingly superluminal speeds at quantum entanglement as will now be discussed.

In the limit when the hyperbolas in the Minkowski diagram degenerate to light rays, time along such a light ray will thus appear frozen as observed from a stationary system, and as illustrated in Figure 5. Furthermore - and this is the important point - space along such a light ray $(x, x)=(t, t)$ in the stationary system will then coalesce into a point (a "photon”) in the light ray's own system.

This thus also has a bearing on the problem of waveparticle duality, and thus explains how the wave function for a light ray from a source somewhere, say from far away back in the past, and going to somewhere, say far away into the future, can seem to completely vanish and simply coalesce into a photon just by being observed. 
The reason for this behaviour is thus that whereas the light ray has a possibly infinite extension in the stationary system, in its own co-moving system it is just a point as calculated by the Lorentz transformation and illustrated in Figure 5. If the photon at this point in its own system then suddenly disappears, e $g$ by being absorbed by a detector, then obviously the entire light ray as observed in the stationary system will also disappear, even though it would seem to extend from infinity to infinity.

Two entangled photons, each one of which is travelling along such a light ray in the stationary system, have then similarly coalesced to be always simultaneously at the same point in their own system as is schematically depicted in Figure 6. Thus if one photon of such an entangled pair is detected in one state, then the other photon in the pair is simultaneously detected at the same point in their own system in the corresponding other state. No matter how distant they may be from each other in the stationary system, in their own system the two entangled photons are both at every instant simultaneously at the same point - until each may be observed somewhere in the stationary system. In their own system, there is as discussed above thus no spacelike, nor timelike separation between the entangled photons, as has also been verified in recent experiments $[18,19]$.

In order for the entangled photons to show this kind of seemingly superluminal interaction between them, they first need to have propagated relative to each other with the speed of light in the stationary system. So in this first stage there is thus no superluminal velocities involved. In the second stage, when they seemingly interact instantaneously despite the possibly large distance separating them in the stationary system, then this is due to exactly the same property of light that causes an entire light beam to disappear and coalesce into a photon when observed as discussed above.

In the above explanation, the entangled quanta were considered to move with the velocity of light in order for their space and time coordinates to coalesce into a point in their own system as described above. The same would obviously be approximately true also for other particles than photons if they just move with velocities close enough to the speed of light (or otherwise along hyperbolic trajectories as in Figure 5, in which case the above argument would still apply).

\section{Concluding Remarks}

In this paper, three phenomena - the evanescent field, quantum tunnelling and quantum entanglement - have been studied where superluminal velocities might possibly be involved. In all three cases there is an initial disturbance that sets the stage and opens a channel for the later, possible superluminal propagation. This initial disturbance propagates with the velocity of light in accordance with the special theory of relativity. Once this stage is set, it seems as if all three candidates can involve what may appear as superluminal propagation of some kind along this channel that thus has been opened.

However, at this later stage the seemingly superluminal propagation may then be due just to a superluminal group velocity, which actually may very well exceed the speed of light, as illustrated by other examples of such arbitrarily large group velocities, such as cutting scissors or light from a rotating beacon or a rotating pulsar at large distances.

Superluminal velocities in phenomena in nature are thus a reality, but are most likely to turn out to be merely due to superluminal group velocities and still be within the realm of special relativity - even though they may, as in the case of quantum entanglement, all the same seem quite paradoxical.

\section{Acknowledgement}

The author is indebted to Dr Hans-Olov Zetterström for many fruitful discussions and valuable suggestions with regard to this study.

\section{References}

[1] A. Aspect, P. Grangier, and G. Roger, "Experimental Realization of Einstein-Podolsky-Rosen-Bohm Gedanken experiment: A New Violation of Bell's Inequalities”, Phys. Rev. Lett., 49, 91-94 (1982)

[2] J. S. Bell, Speakable and unspeakable in quantum mechanics (Cambridge University Press, 1987) pp. 142, 144.

[3] A. Bergstrom, “Optoelectronic circuit element”, US Patent 4,254,333 (1981).

[4] A. Bergstrom, C. Dahlstrom, "Sensor-Taste nach optischen Prinzip”, Elektronik Industrie 12, 70-71 (1984).

[5] A. Bergstrom, "Relativistic invariance and the expansion of the universe”, Nuovo Cimento 27B, 145-160 (1975).

[6] A. Bergstrom, "Lorentz-covariant quantum transport and the origin of dark energy”, Phys. Scr. 83, 055901 (2011).

[7] A. Bergstrom, "Big Crunch, Big Rip - or a self-similar expansion replenished by dark matter and dark energy?”, Int. Journal of Physics 2, 146-150 (2014).

[8] J. L. Borges, Kafka and his precursors, in Labyriths (Penguin, 2000), p 234.

[9] A. M. Steinberg, P. G. Kwiat, and R. Y. Chiao, "Measurement of the single-photon tunnelling time”, Phys. Rev. Lett. 71, 708 (1993).

[10] A. P. L. Barbero, H. E. Hernández-Figueroa, E. Recami, "Propagation speed of evanescent modes", Phys. Rev. E 62, 86288635 (2000).

[11] R. Y. Chiao, P. G. Kwiat, and A. M. Steinberg, "Faster than light?”, Scientific American, Aug 1993, 52-60.

[12] M. Arndt, O. Nairz, J. Vos-Andreae, et al., "Wave-particle duality of C60 molecules”, Nature 401, 680-682 (1999).

[13] K. C. Lee, M. R. Sprague, B. J. Sussman, et al., "Entangling macroscopic diamonds at room temperature", Science 334 12531256 (2011).

[14] H. Zbinden, J. Brendel, N. Gisin, W. Tittel, "Experimental test of nonlocal quantum correlations in relativistic configurations", Phys. Rev. A 63, 22111 (2001).

[15] S. R. Mainwaring, G. E. Stedman, “Accelerated clock principles in special relativity”, Phys. Rev. A 47, 3611-3619 (1993).

[16] G. Barton, Introduction to the relativity principle (Wiley, 1999), Ch 8.

[17] A. Bergstrom, "Can time in special relativity appear frozen despite the clock hypothesis says it cannot?”, Int. Journal of Physics 1, 146-150 (2013).

[18] X.-S. Ma, S. Zotter, J. Kofler, R. Ursin, T. Jennewein, Č. Brukner, and A. Zeilinger, "Experimental delayed-choice entanglement swapping", Nature Physics 8, 480-485 (2012).

[19] E. Megidish, A. Halevy, T. Shacham, T. Dvir, L. Dovrat, and H. S. Eisenberg, "Entanglement swapping between photons that have never coexisted", Phys. Rev. Lett. 110, 210403 (2013). 